Research Article

\title{
Electronic Structure and Magnetic Coupling of Pure and Mg-Doped $\mathrm{KCuF}_{3}$
}

\author{
Fausto Cargnoni, ${ }^{1}$ Simone Cenedese, ${ }^{1}$ Paolo Ghigna $\left({ }^{2}{ }^{2}\right.$ \\ Mario Italo Trioni, ${ }^{1}$ and Marco Scavini $\mathbb{D i D}^{1,3}$ \\ ${ }^{1}$ Consiglio Nazionale delle Ricerche, Istituto di Scienze e Tecnologie Molecolari, Via Golgi 19, 20133 Milano, Italy \\ ${ }^{2}$ Dipartimento di Chimica, Università di Pavia and Unità INSTM di Pavia, Viale Taramelli 13, 27100 Pavia, Italy \\ ${ }^{3}$ Dipartimento di Chimica, Università degli Studi di Milano, Via Golgi 19, 20133 Milano, Italy \\ Correspondence should be addressed to Marco Scavini; marco.scavini@unimi.it
}

Received 23 January 2018; Revised 18 June 2018; Accepted 3 July 2018; Published 15 August 2018

Academic Editor: Da-Ren Hang

Copyright (C) 2018 Fausto Cargnoni et al. This is an open access article distributed under the Creative Commons Attribution License, which permits unrestricted use, distribution, and reproduction in any medium, provided the original work is properly cited.

\begin{abstract}
We investigated the electronic and magnetic properties of $\mathrm{KCuF}_{3}$ and $\mathrm{KCu}_{0.875} \mathrm{Mg}_{0.125} \mathrm{~F}_{3}$ crystals by means of Density Functional periodic computations at the B3LYP level of theory. We considered four possible magnetic ordering of the unpaired electrons on copper ions. Both materials are correctly predicted as being $1 \mathrm{D}$ antiferromagnetic insulators, and the superexchange parameters in the crystallographic $a b$ planes and along the $c$ direction measure +10 and $-600 \mathrm{~K}$, respectively. Residual spin polarization is found also on fluorine atoms, in agreement with literature results. We found a complete orbital ordering at $\mathrm{Cu}$ sites: in the copper reference frame $\mathrm{d}_{\mathrm{xy}}, \mathrm{d}_{\mathrm{yz}}, \mathrm{d}_{\mathrm{xz}}$, and $\mathrm{d}_{\mathrm{z}^{2}}$ orbitals contain about 2 electrons each, while the $\mathrm{d}_{\mathrm{x}^{2}-\mathrm{y}^{2}}$ orbital is only partially filled. The perturbation induced by doping of $\mathrm{KCuF}_{3}$ with $\mathrm{Mg}$ is very strong and localized on the first shell of $\mathrm{F}$ neighbours. $\mathrm{Mg}$ has a very small influence on the ordering of the $3 \mathrm{~d}$ orbitals of copper and on the $\mathrm{Cu}$-Cu magnetic superexchange parameters but reduces significantly the absolute energy differences between the antiferromagnetic ground state and the ferromagnetic phase, in agreement with the experiment. The absence of long range effects makes $\mathrm{Mg}$ a suitable dopant for the investigation of strongly correlated electronic systems by means of orbital dilution.
\end{abstract}

\section{Introduction}

Strong correlation of electrons in solids leads to a variety of phenomena that are still very far to be really understood. They range from superconductivity in cuprates, to colossal magnetoresistance in manganites, to heavy fermions formation in rare earth intermetallics. It is widely recognized that one of the reasons for the lack of understanding of these systems is that several degrees of freedom are intimately interconnected and that the identification of the leading interaction is a challenging task, a typical example being the interplay between orbital ordering (OO) and cooperative Jahn-Teller distortion (cJTd). As pointed out by Kugel and Khomskii in their seminal work [1], in presence of strong electron correlation orbitals are subject to exchange interaction and a preexisting $\mathrm{OO}$ tends to amplify any lattice instability. In this scenario, $\mathrm{OO}$ is the leading interaction that causes the setting up of cJTd. The pseudocubic perovskite $\mathrm{KCuF}_{3}$ has always been considered as a model system for testing the KugelKhomskii model. However, the argument is still a matter of debate. LDA + DMFT calculations [2], for example, showed that the superexchange mechanisms in $\mathrm{KCuF}_{3}$ are not strong enough to stabilize the cJTd up to the high temperature regime where it is experimentally still found to survive. While orbital degrees of freedom are not directly accessible in this system, the resonant X-ray Scattering (RXS) signal is dominated by the distortions in the $\mathrm{F}^{-}$ion positions $[3,4]$, and melting of cJTd has been observed in perovskite samples of composition $\mathrm{KCu}_{1-\mathrm{x}} \mathrm{Mg}_{\mathrm{x}} \mathrm{F}_{3}$, with $T$ increasing linearly at decreasing $x[5,6]$. When extrapolated to $x=0$, a value of $0.166 \mathrm{eV}$ is found, which can be considered as a reasonable estimate of the cJTd energy in the pure $\mathrm{KCuF}_{3}$ compound. This experimental result is in nice agreement with the LDA + DMFT calculations [2] and supports the conclusion 
that the electron phonon coupling is the most important interaction in stabilizing the peculiar structure of $\mathrm{KCuF}_{3}$. The subtle interplay between spin, orbital, and vibrational degrees of freedom of this material is still the subject of experimental and theoretical investigations [7-18], adopting a variety of techniques ranging from inelastic Raman and $\mathrm{X}$-rays scattering and absorption, [11, 13], neutron scattering $[10,14]$, DFT based electronic structure computations [8], and lattice dynamics $[7,15]$ and, more recently, calculations within the variational Green's function formalism $[17,18]$.

With the aim of better understanding the relevance of these experimental findings and to gather them in the wider context of electronic structure of strongly correlated electron systems, here we present an ab initio study of Mg-doped $\mathrm{KCuF}_{3}$. To this purpose, also the electronic and magnetic properties of pure $\mathrm{KCuF}_{3}$ are investigated and discussed in comparison with the doped system and with previous calculations. Even for the pure compound, we present an accurate characterization of all the four possible magnetic structures, which has never been previously discussed in detail. However, the main target of our investigation is to characterize the structural, electronic, and magnetic properties of $\mathrm{Mg}$-doped $\mathrm{KCuF}_{3}$ and to determine how $\mathrm{Mg}$ doping affects the properties of this material.

The paper is organized as follows: in the next section we present the details of our computations, along with a brief excursion on the atomistic arrangement and the energetic in pure and $\mathrm{Mg}$-doped $\mathrm{KCuF}_{3}$. Then we discuss the electronic structure of these materials, using either the typical instruments of solid state physics and the Quantum Theory of Atoms in Molecules (QTAIM), which is based on a direct space analysis of the electron density distribution and provides a description closest to the chemists' point of view. Third, we present the magnetic coupling parameters obtained for the pure and the Mg-doped samples. The last section contains a general discussion about the effect of $\mathrm{Mg}$ doping in $\mathrm{KCuF}_{3}$ together with conclusions and perspectives.

\section{Computational Details and Structure Optimization}

The electronic structure and magnetic coupling of pure and $\mathrm{Mg}$-doped $\mathrm{KCuF}_{3}$ have been determined by means of first principles periodic computations, as implemented in the CRYSTAL [19] code. For most computations we adopted the unrestricted B3LYP Hamiltonian, which proved to be successful in describing low-dimensional magnetic copper insulators [20]. Several other exchange-correlation functionals have been selected to perform different tests. The electronic wavefunctions have been expanded in terms of all electrons Gaussian basis sets designed for solid state computations. We assigned to potassium atoms a $21 \mathrm{~s} 13 \mathrm{p} 3 \mathrm{~d}$ basis set contracted to $5 \mathrm{~s} 4 \mathrm{pld}$ [21], to copper a $21 \mathrm{~s} 13 \mathrm{p} 5 \mathrm{~d}$ set contracted to $6 \mathrm{~s} 5 \mathrm{p} 2 \mathrm{~d}$ [22], to fluorine a $12 \mathrm{~s} 5 \mathrm{p}$ set contracted to $4 \mathrm{~s} 3 \mathrm{p}$ [23], and to magnesium a $15 \mathrm{~s} 7 \mathrm{pld}$ set contracted to 4s3pld [24]. To obtain the wavefunctions of four different magnetic phases of $\mathrm{KCuF}_{3}$, in each computation we locked properly the atomic spin of copper atoms during the first 30 cycles of the iterative procedure within the CRYSTAL code,

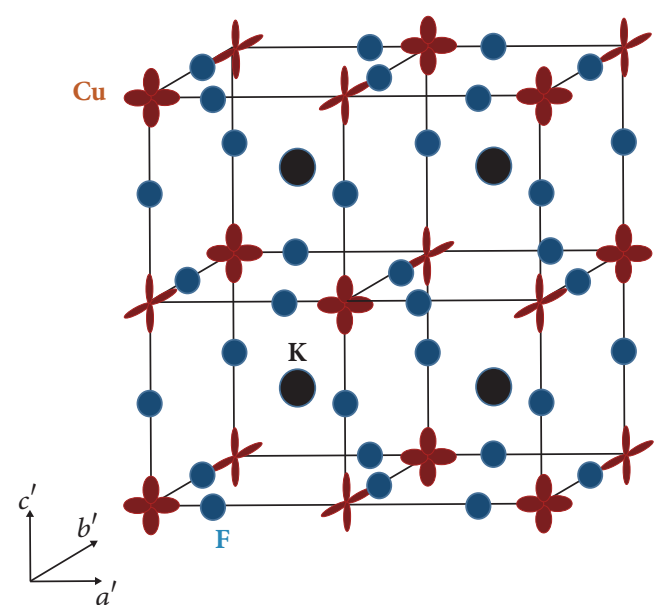

FIgURE 1: Stick-and-ball representation of $\mathrm{KCuF}_{3}$ in the $\mathrm{Pmmm}$ space group (see text for details). For the sake of clarity, only half a cell is shown in the figure $(0 \leq y \leq 0.5)$. The shifts of the position of $\mathrm{F}$ ions in the ab plane, in respect to the middle of the $\mathrm{Cu}-\mathrm{Cu}$ internuclear axis as in cubic perovskite, are enlarged to highlight the deformation of $\mathrm{CuF}_{6}$ octahedra. The partially filled $\mathrm{d}_{\mathrm{x} 2-\mathrm{y} 2}$ orbitals responsible for orbital ordering are schematically represented in red.

TABLE 1: Crystallographic parameters of pure KCuF3 in the AF1 magnetic phase (I4/mcm space group).

\begin{tabular}{lccc}
\hline Reference & $a, b(\AA)$ & $c(\AA)$ & $\mathrm{x}_{\mathrm{F}}$ \\
\hline this work & 5.92 & 7.96 & 0.230 \\
Experiment [25] & $5.8569(6)$ & $7.8487(8)$ & $0.2276(1)$ \\
\hline
\end{tabular}

and then we let the wavefunction free to evolve towards the converged solution. The four magnetic phases considered in this study are all the possible combinations of ferromagnetic (FM) and antiferromagnetic (AF) order among neighbouring $\mathrm{Cu}$ atoms pairs in the $a b$ plane and along the $c$ axis (see Figure 1). In particular, in the AF1 phase there is AF order along the $c$ axis and FM order in the $a b$ plane. The AF2 phase presents FM order along the $c$ axis and AF order in the $a b$ plane. In the AF3 and in the F phases the order is entirely AF and FM, respectively.

As a first step, we optimized the structure of the AF1 phase of pure $\mathrm{KCuF}_{3}$ within the $I 4 / \mathrm{mcm}$ space group. This corresponds to the optimization of two cell parameters $(a=b$, $c$ ), and of the relative position of fluorine atoms $\left(\mathrm{x}_{\mathrm{F}}\right)$ on the internuclear axis of $\mathrm{Cu}-\mathrm{Cu}$ neighbours in $a b$ planes. The resulting data are reported in Table 1, and they are in fair agreement with the experimental results, relative errors in the estimate of $a$ and $c$ being about 1-1.5\% [25]. The optimized position of $\mathrm{F}$ atoms $\left(\mathrm{x}_{\mathrm{F}}=0.230\right)$ confirms that in the AF1 phase copper is surrounded by a distorted octahedron of fluorine atoms. The optimized $\mathrm{Cu}-\mathrm{F}$ distances in $a b$ planes are 1.93 and $2.26 \AA$ and $1.99 \AA$ along the $c$ axis.

To compute the wavefunction of the four magnetic phases of pure and $\mathrm{Mg}$-doped $\mathrm{KCuF}_{3}$, we defined a supercell in the Pmmm space group. The new cell vectors are defined as $\boldsymbol{a}^{\prime}=\boldsymbol{a}+\boldsymbol{b}$, $\boldsymbol{b}^{\prime}=\boldsymbol{a}-\boldsymbol{b}$, and $\boldsymbol{c}^{\prime}=\boldsymbol{c}$, and the arrangement of the atoms within this choice is reported in Figure 1. Our calculations confirm that 
the $\mathrm{AFl}$ phase is the ground state for pure $\mathrm{KCuF}_{3}$. The $\mathrm{AF} 3$ phase, where the FM interactions in $a b$ planes are turned into $\mathrm{AF}$ ones, presents a very small increase in energy, with $\mathrm{E}_{\mathrm{AF}_{3}}$ $\mathrm{E}_{\mathrm{AF1}}=12 \mathrm{meV} /$ cell. A more marked energy increase is found when the AF interactions along the $c$ axis are turned into FM ones: $\mathrm{E}_{\mathrm{AF} 2}-\mathrm{E}_{\mathrm{AF} 1}=229 \mathrm{meV} / \mathrm{cell}$, and $\mathrm{E}_{\mathrm{F}}-\mathrm{E}_{\mathrm{AF1}}=234 \mathrm{meV} /$ cell.

Doping of $\mathrm{KCuF}_{3}$ with $\mathrm{Mg}$ atoms is described as a regular substitution of a single copper atom in each supercell, which corresponds to the overall composition of $\mathrm{KCu}_{0.875} \mathrm{Mg}_{0.125} \mathrm{~F}_{3}$. To account for the geometrical distortions introduced by $\mathrm{Mg}$ in the crystal network, we optimized the structure of the AF1 phase of Mg-doped $\mathrm{KCuF}_{3}$. We optimized the cell parameters $a^{\prime}\left(=b^{\prime}\right)$ and $c^{\prime}$, as well as the position of the 6 fluorine atoms closer to $\mathrm{Mg}$. Though in principle a much larger set of coordinates should be considered to perform a complete optimization of the structure, we are confident that the perturbation induced in the electronic properties by $\mathrm{Mg}$ doping is properly accounted for by considering just the distortion of the first shell of neighbours surrounding the doping site. This assumption is strengthened by the results obtained as discussed in the following, as the perturbation induced by $\mathrm{Mg}$ doping is indeed very short-ranged. The geometrical parameters obtained for the AFl phase have been adopted also for the AF2, AF3, and F magnetic arrangements. Test computations for the AF1 phase, conducted on a larger supercell, proved that no noticeable displacements occur for other atoms than the first fluorine neighbours of $\mathrm{Mg}$.

The doping of $\mathrm{KCuF}_{3}$ with a $12.5 \%$ percentage of $\mathrm{Mg}$ has no relevant effect on the cell parameters, at variance with experimental findings [26]. The dopant element reduces the distortion of the surrounding fluorine octahedron, and the three unique $\mathrm{Mg}-\mathrm{F}$ distances measures are 1.94 and $2.20 \AA$ in $a b$ planes and $1.98 \AA$ along $c$. It should be noted that in our calculations each $\mathrm{Mg}$ ion is surrounded by six $\mathrm{Cu}$ ions, while in real samples clusters of dopant ions can be formed, and they may affect the cell parameters in a more effective manner. However, the investigation of complex defect structures is beyond the scope of the present paper.

The changes of the $\mathrm{Mg}$-F distances with respect to the corresponding $\mathrm{Cu}-\mathrm{F}$ ones in the undoped material clearly reflect also on the environment of the neighbouring $\mathrm{Cu}$ atoms, and this has the effect of reducing also the distortions of the fluorine octahedra surrounding copper. Noticeably, Mg doping does not alter the ordering in energy of the four magnetic phases considered, but the energy differences become significantly smaller: $\mathrm{E}_{\mathrm{AF} 3}-\mathrm{E}_{\mathrm{AF1}}=4 \mathrm{meV} /$ cell, $\mathrm{E}_{\mathrm{AF} 2}-\mathrm{E}_{\mathrm{AF1}}=$ $168 \mathrm{meV} /$ cell, and $\mathrm{E}_{\mathrm{F}}-\mathrm{E}_{\mathrm{AF1}}=184 \mathrm{meV} / \mathrm{cell}$. The steep decrease of the energy difference between the AF1 and the F phases is in agreement with experimental determinations [26].

\section{Electronic Structure}

A very useful approach to get insight into the chemistry of a given material is represented by the Quantum Theory of Atoms in Molecules. This theory is based on the real space analysis of the electron density distribution, as a way to uniquely define the atomic and bonding properties of a given chemical system. The basic notions of this theory, several applications of the QTAIM to solid state systems, and the formalism adopted by the authors can be found in [27-29]. The QTAIM computations have been carried out using the TOPOND code [30], interfaced with CRYSTAL [19]. Figures 2 and 3 report the electron density, the negative of the Laplacian of the electron density, and the spin density in selected crystallographic planes, while we collected in Table 2 the properties of $\mathrm{Cu}-\mathrm{F}$ bonds in pure $\mathrm{KCuF}_{3}$, along with the $\mathrm{Cu}-\mathrm{F}$ bonding properties in $\mathrm{KCu}_{0.875} \mathrm{Mg}_{0.125} \mathrm{~F}_{3}$ for the three unique copper atoms closer to $\mathrm{Mg}$ (see also Figure 4). We reported just the results obtained for the AF1 phase, because no relevant change in $\mathrm{Cu}-\mathrm{F}$ bonds occurs when the magnetic ordering of spins changes. Data in Table 2 are typical of interactions between closed shells, confirming that $\mathrm{Cu}-\mathrm{F}$ bonds are indeed dominated by the large charge transfer from copper to fluorine (assuming the neutral atoms as reference). The value of the electron density at the bond critical point (bcp) is always small. The Laplacian at the $b c p$ is large and positive, and hence it is dominated by its positive eigenvalue $\lambda_{3}$ corresponding to the curvature along the $\mathrm{Cu}$ $\mathrm{F}$ internuclear axis. The $b c p$ of $\mathrm{Cu}-\mathrm{F}$ falls into a region of charge depletion; i.e., the electron density is not accumulated on the $\mathrm{Cu}-\mathrm{F}$ axis but is removed from it, which is typical of interactions between closed shells. The differences among the $\mathrm{Cu}-\mathrm{F}$ bonds can be entirely attributed to the different $\mathrm{Cu}-\mathrm{F}$ internuclear distances: as Cu-F lengthens, $\rho_{\mathrm{b}}, \nabla^{2} \rho_{\mathrm{b}}$, and $\lambda_{3 \mathrm{~b}}$ decrease in absolute value. We observed no change in the nature of $\mathrm{Cu}-\mathrm{F}$ bonds due to the presence of $\mathrm{Mg}$ into the lattice, and also in this case the variations of the bonding properties are strictly related to the changes of the internuclear $\mathrm{Cu}-\mathrm{F}$ distance.

The atomic properties of pure $\mathrm{KCuF}_{3}$ according to the QTAIM are collected in Table 3. We first note that the charge of copper atoms deviates significantly from the formal value of $+2 \mathrm{e}$, while the charge of potassium is quite close to $+1 \mathrm{e}$. Accordingly, the charge of fluorine atoms is far from the formal value of $-1 \mathrm{e}$, and its value is $-0.76 \mathrm{e}$ for $\mathrm{F}$ atoms along the $c$ axis and $-0.78 \mathrm{e}$ for $\mathrm{F}$ atoms in $a b$ planes. This indicates that the material is not fully ionic.

The local distribution of electronic density within the atomic basins can be rationalized analyzing its traceless quadrupole moment tensor, Q. The eigenvectors of $\mathrm{Q}$ associated with negative (positive) eigenvalues correspond to the directions of charge accumulation (depletion) with respect to an ideal spherical distribution. In pure and $\mathrm{Mg}$-doped $\mathrm{KCuF}_{3}$ potassium ions are nearly spherical, and the eigenvalues of $\mathrm{Q}$ are very small. Conversely, the charge distribution in the atomic basins of copper atoms presents a large anisotropy. The electron density is preferentially accumulated along the long $\mathrm{Cu}-\mathrm{F}$ bonds at $2.26 \AA$, with the corresponding eigenvalue of $\mathrm{Q}$ that measures about -1 , and it is removed from the directions of the shorter $\mathrm{Cu}-\mathrm{F}$ bonds at 1.93 and $1.99 \AA$, with the eigenvalues of $\mathrm{Q}$ that measure $+0.36(\mathrm{Cu}-\mathrm{F}(c)$ at $1.99 \AA)$ and $+0.69(\mathrm{Cu}-\mathrm{F}(a b)$ at $1.93 \AA)$. As already observed in the literature [29-31], there is a nice agreement between orbital view and quadrupole moment tensor analysis in systems containing transition metals compounds. Consistently, in our material both approaches predict that the electron density of copper is removed from the axes of the short $\mathrm{Cu}-\mathrm{F}$ bonds and accumulated along the longest bonds. 
TABLE 2: $\mathrm{Cu}-\mathrm{F}$ bonding properties in the $\mathrm{AFl}$ phase of pure and $\mathrm{Mg}$-doped $\mathrm{KCuF}_{3}$.

\begin{tabular}{|c|c|c|c|c|c|}
\hline System & bond & distance $^{\mathrm{a}}$ & $\rho_{\mathrm{b}} \cdot 10^{2 \mathrm{~b}, \mathrm{c}}$ & $\nabla^{2} \rho_{\mathrm{b}} \cdot 10^{2 \mathrm{~b}, \mathrm{~d}}$ & $\lambda_{3 \mathrm{~b}} \cdot 10^{2 \mathrm{~b}, \mathrm{e}}$ \\
\hline \multirow[t]{3}{*}{$\mathrm{KCuF}_{3}$} & $\mathrm{Cu}-\mathrm{F}(a b)$ & 1.93 & 7.8 & 51 & 71 \\
\hline & $\mathrm{Cu}-\mathrm{F}(a b)$ & 2.26 & 3.7 & 15 & 23 \\
\hline & $\mathrm{Cu}-\mathrm{F}(c)$ & 1.99 & 6.5 & 41 & 58 \\
\hline \multirow[t]{9}{*}{$\mathrm{KCu}_{0.875} \mathrm{Mg}_{0.125} \mathrm{~F}_{3}$} & $\mathrm{Cu}(\mathrm{a})-\mathrm{F}(a b)$ & 1.99 & 6.7 & 41 & 58 \\
\hline & $\mathrm{Cu}(\mathrm{a})-\mathrm{F}(a b)$ & 2.26 & 3.7 & 15 & 23 \\
\hline & $\mathrm{Cu}(\mathrm{a})-\mathrm{F}(\mathrm{c})$ & 1.99 & 6.5 & 40 & 57 \\
\hline & $\mathrm{Cu}(\mathrm{b})-\mathrm{F}(a b)$ & 1.93 & 7.7 & 51 & 71 \\
\hline & $\mathrm{Cu}(\mathrm{b})-\mathrm{F}(a b)$ & 2.25 & 3.8 & 16 & 24 \\
\hline & $\mathrm{Cu}(\mathrm{b})-\mathrm{F}(\mathrm{c})$ & 1.99 & 6.5 & 41 & 58 \\
\hline & $\mathrm{Cu}(\mathrm{c})-\mathrm{F}(a b)$ & 1.93 & 7.8 & 51 & 71 \\
\hline & $\mathrm{Cu}(\mathrm{c})-\mathrm{F}(a b)$ & 2.26 & 3.7 & 15 & 23 \\
\hline & $\mathrm{Cu}(\mathrm{c})-\mathrm{F}(\mathrm{c})$ & 2.00 & 6.4 & 40 & 56 \\
\hline
\end{tabular}

${ }^{a}$ Values in $\AA ;{ }^{b}$ the subscript $b$ indicates that the properties are evaluated at the bond critical point; ${ }^{c} \rho$ is the electron density; ${ }^{\mathrm{d}} \nabla^{2} \rho$ is the Laplacian of the electron density; ${ }^{\mathrm{e}} \lambda_{3}$ is the positive eigenvalue of the Hessian of the electron density.

TABLE 3: Atomic properties in pure $\mathrm{KCuF}_{3}$ computed with the QTAIM approach.

\begin{tabular}{|c|c|c|c|c|}
\hline System & Atom & $\mathrm{q}^{\mathrm{a}}$ & $\mathrm{V}^{\mathrm{b}}$ & $Q^{c}$ \\
\hline \multirow[t]{12}{*}{ AF1 } & $\mathrm{K}$ & 0.93 & 124 & -0.02 \\
\hline & & & & 0.01 \\
\hline & & & & 0.01 \\
\hline & $\mathrm{Cu}$ & 1.38 & 61 & -1.05 \\
\hline & & & & 0.36 \\
\hline & & & & 0.69 \\
\hline & $\mathrm{F}(c)$ & -0.76 & 93 & -0.49 \\
\hline & & & & -0.49 \\
\hline & & & & 0.97 \\
\hline & $\mathrm{F}(a b)$ & -0.78 & 96 & -0.44 \\
\hline & & & & -0.41 \\
\hline & & & & 0.85 \\
\hline \multirow[t]{3}{*}{ AF2 } & $\mathrm{Cu}$ & 1.39 & 61 & -1.00 \\
\hline & & & & 0.28 \\
\hline & & & & 0.72 \\
\hline \multirow[t]{3}{*}{ AF3 } & $\mathrm{Cu}$ & 1.39 & 61 & -1.06 \\
\hline & & & & 0.36 \\
\hline & & & & 0.69 \\
\hline \multirow[t]{3}{*}{$\mathrm{F}$} & $\mathrm{Cu}$ & 1.39 & 61 & -1.02 \\
\hline & & & & 0.33 \\
\hline & & & & 0.69 \\
\hline
\end{tabular}

${ }^{\mathrm{a}}$ Atomic charge (electrons); ${ }^{\mathrm{b}}$ atomic volume $\left(\mathrm{bohr}^{3}\right) ;{ }^{\mathrm{c}}$ eigenvalues of the traceless quadrupole moment tensor (a.u.); positive (negative) values indicate that the electron density is preferentially removed (accumulated) along the direction of the corresponding eigenvector. The first eigenvalue for $\mathrm{Cu}$ atoms corresponds to the direction of the longest $\mathrm{Cu}-\mathrm{F}$ bonds.

Quite interestingly, also the charge distribution of $\mathrm{F}$ anions is significantly anisotropic. The positive eigenvalues of $\mathrm{Q}(+0.97$ for $\mathrm{F}(c),+0.85$ for $\mathrm{F}(a b))$ indicate that fluorine atoms remove electron density from the $\mathrm{Cu}-\mathrm{F}$ bonding direction and accumulate charge away from copper neighbours. As can be seen in Table 3, copper atoms behave similarly in all the magnetic phases considered, and the same is found also for the other atoms of the crystals. The very small variations of the atomic properties observed among the four magnetic phases considered do not follow any recognizable trend.

The insertion of $\mathrm{Mg}$ atoms in the structure induces a strong local perturbation. In fact, the QTAIM charge of $\mathrm{Mg}$ is about $+1.77 \mathrm{e}$, significantly larger as compared to copper, and hence about $0.4 \mathrm{e}$ is delivered in the structure. Furthermore, the atomic volume of $\mathrm{Mg}$ is $33 \mathrm{bohr}^{3}$, much smaller than the value of $61 \mathrm{bohr}^{3}$ exhibited by Cu. Finally, the distribution of the electron density in the atomic basin of $\mathrm{Mg}$ atoms is almost spherical, with eigenvalues of $\mathrm{Q}$ comparable to those of $\mathrm{K}$ atoms.

The strong perturbation due to $\mathrm{Mg}$ doping is nearly entirely counterbalanced by the six fluorine atoms closer to $\mathrm{Mg}$. The electron population of fluorine atoms increase by $0.10,0.07$, and 0.04 e for $F$ neighbours at $1.93,1.98$, and $2.20 \AA$, respectively. The atomic volumes increase accordingly: $+6 \mathrm{bohr}^{3}$ for $\mathrm{F}$ atoms at 1.93 and $+5 \mathrm{bohr}^{3}$ and $+4 \mathrm{bohr}^{3}$ for $\mathrm{F}$ atoms at 1.98 and $2.20 \AA$. It is worth noting that also the distribution of electron density within the atomic basins of $\mathrm{F}$ atoms close to $\mathrm{Mg}$ becomes less anisotropic: the positive eigenvalue of $\mathrm{Q}$ for $\mathrm{F}$ neighbours in $a b$ planes decreases from about +0.85 in the pure sample to about +0.55 in the doped compound and from +0.97 to +0.71 for $F$ neighbours along the $c$ axis. No relevant change in the atomic properties of other atoms is observed.

A complementary view to the real space description of the electron density distribution is given by the band structure and the analysis of the Density of States (DOS) obtained by the band structure. First of all, at the B3LYP level of theory our computations correctly predict that the system is an insulator whatever the magnetic phase considered, and $\mathrm{Mg}$ doping does not alter significantly the band gap. The system remains nonconducting even imposing an orbitally ordered solution to the undistorted cubic structure. Figure 1 of the SI section confirms that the valence states of pure and $\mathrm{Mg}$ doped distorted $\mathrm{KCuF}_{3}$ comes from a partial hybridization 
$\rho(r)$
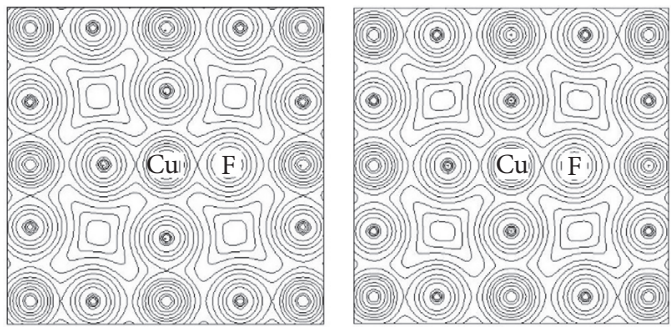

$-\nabla^{2} \rho(r)$

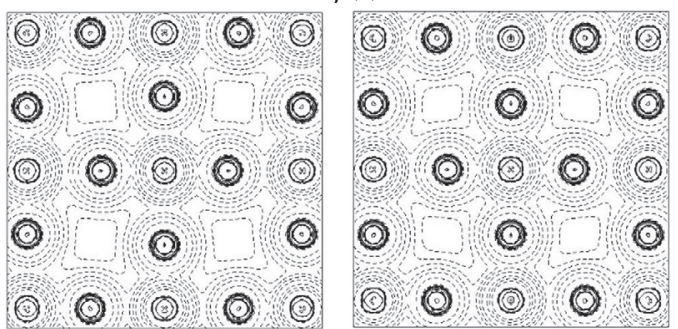

$\operatorname{spin} \operatorname{density}(\alpha-\beta)$
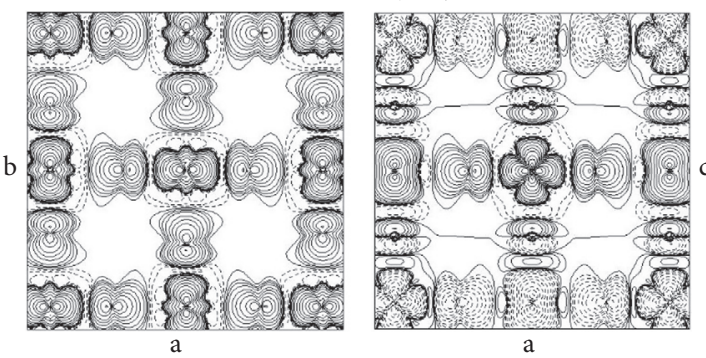

FIGURE 2: Contour plots of the electron density, the Laplacian of the electron density changed in sign and spin density in pure $\mathrm{KCuF}_{3}$. Left panels refer to the $a b$ and the right panels to the $a c$ plane. Full and dashed lines indicate positive and negative values of the plotted function, respectively. Plots refer to the AF1 phase.

between copper and fluorine orbitals, consistently with the picture of the mixed covalent-ionic character of $\mathrm{Cu}-\mathrm{F}$ bonds, as evidenced above by QTAIM analysis. The contribution of fluorine orbitals to valence states becomes relevant at $2.25 \mathrm{eV}$ below the Fermi level and increases moving to states at even lower energies. The $\mathrm{Mg}$ and $\mathrm{K}$ atoms act just as electron donors, and their orbitals do not concur to the formation of electronic states in the valence region.

In Figure 5 we reported the DOS projected onto the $\mathrm{d}$ orbitals of copper in the pure crystal with AF1 magnetic ordering. To analyze the population of individual orbitals within the copper $\mathrm{d}$ shell, we applied a rotation of the reference frame such that the $\mathrm{z}$ axis is directed along the longest $\mathrm{Cu}-\mathrm{F}$ bonds at $2.26 \AA$, while $\mathrm{x}$ and $\mathrm{y}$ are aligned to the shorter $\mathrm{Cu}-\mathrm{F}$ bonds. For the sake of clarity, the $\mathrm{d}_{\mathrm{xy}}, \mathrm{d}_{\mathrm{xz}}$, and $\mathrm{d}_{\mathrm{yz}}$ are grouped together in Figure 5 and labelled " $\mathrm{t}_{2 \mathrm{~g}}$ " (black curve) while $\mathrm{d}_{\mathrm{x}^{2}-\mathrm{y}^{2}}$ (blue curve) and $\mathrm{d}_{\mathrm{z}^{2}}$ (red curve) orbitals are drawn separately. Figure SI2 of the SI reports the DOS of individuals " $\mathrm{t}_{2 \mathrm{~g}}$ " orbitals. It is easily seen that the $d$ orbitals of copper are completely filled but for the $d_{x^{2}-y^{2}}$ one, which is empty in just one spin component neglecting a small contribution at about $5 \mathrm{eV}$ below the Fermi level. This
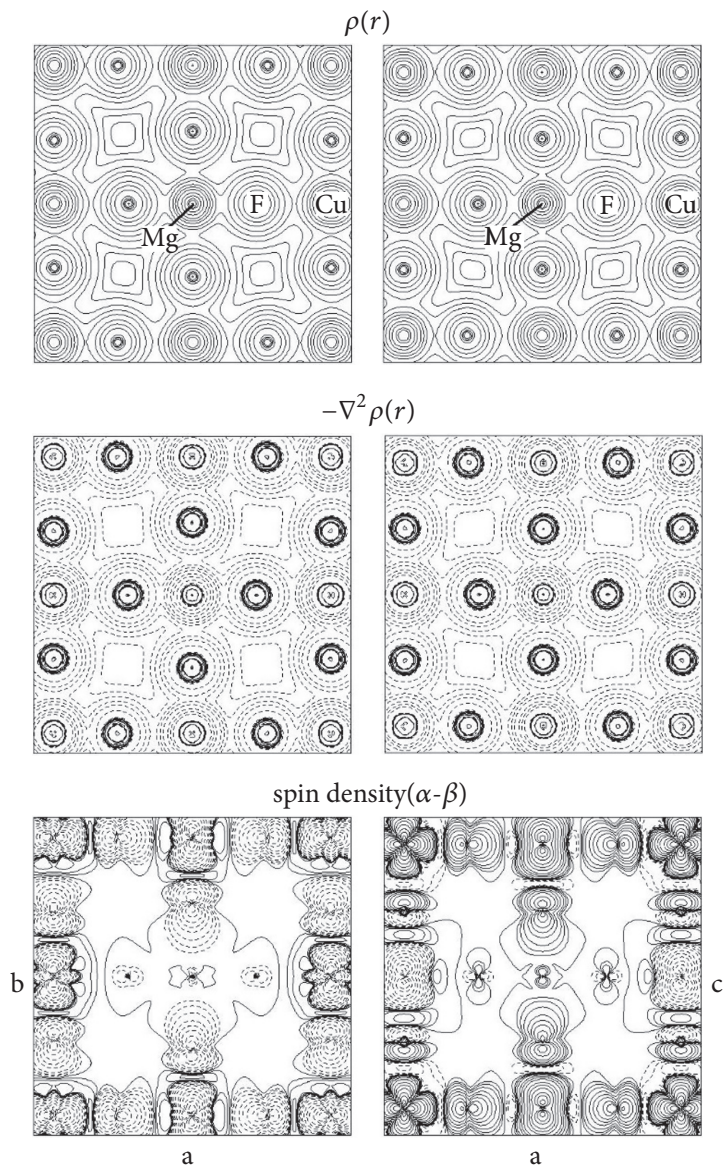

FIgURE 3: Contour plots of the electron density, the Laplacian of the electron density changed in sign and spin density in Mg-doped $\mathrm{KCuF}_{3}$. Left panels refer to the $a b$ and the right panels to the $a c$ plane. Full and dashed lines indicate positive and negative values of the plotted function, respectively. Plots refer to the AF1 phase.

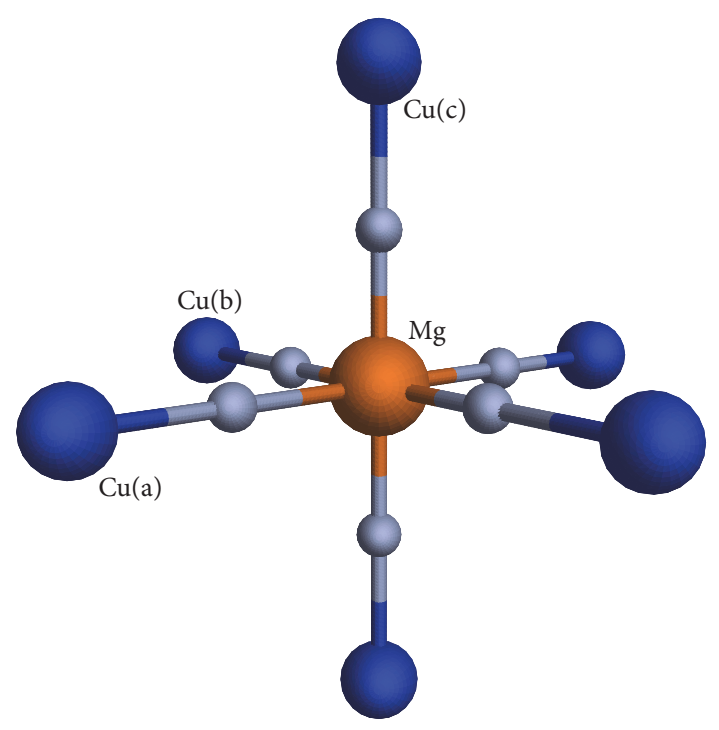

FIGURE 4: Stick-and-ball representation of the atoms surrounding $\mathrm{Mg}$ in the doped compound. $\mathrm{Mg}$ is 2.20 and $1.94 \AA$ from fluorine atoms shared with $\mathrm{Cu}(\mathrm{a})$ and $\mathrm{Cu}(\mathrm{b})$, respectively. 


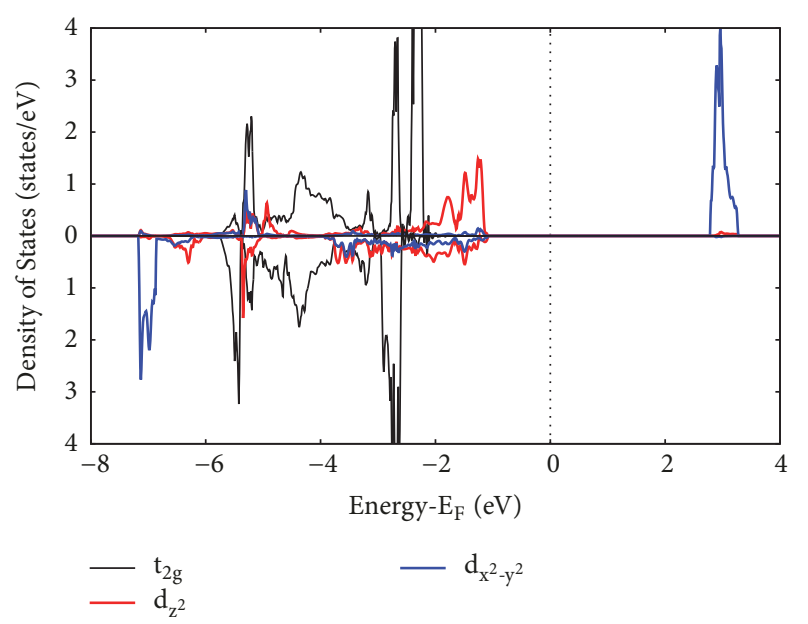

FIgURE 5: Projected density of states on copper atoms of pure $\mathrm{KCuF}_{3}$ determined at the B3LYP level of theory for the AF1 phase. DOS in arbitrary units. Upper and bottom panels refer to different spin components. For the sake of clarity, $\mathrm{d}_{\mathrm{xy}}, \mathrm{d}_{\mathrm{xz}}$, and $\mathrm{d}_{\mathrm{yz}}$ are grouped together and called " $t_{2 g}$ " (black curve) while $d_{x^{2}-y^{2}}$ (blue curve) and $\mathrm{d}_{\mathrm{z}^{2}}$ (red curve) ones are drawn separately.

is consistent with the QTAIM analysis, in particular with the analysis of $\mathrm{Q}$, indicating that copper distributes its electron density preferentially along the axis of the longest $\mathrm{Cu}-\mathrm{F}$ bonds, while the axes of the $\mathrm{Cu}-\mathrm{F}$ intermediate and short bonds are somewhat depleted. Furthermore, this confirms that at this level of theory the system is completely orbitally ordered, consistently with theoretical $[2,20,32-34]$ and experimental $[35,36]$ results for this system. We further note that the DOS coming from $t_{2 g}$ orbitals $\left(d_{x y}, d_{x z}\right.$, and $\left.d_{y z}\right)$ is mainly concentrated in sharp peaks, while the $d_{z^{2}}$ one is closer to the Fermi level and distributed onto a wider range of energies. This picture does not change when different magnetic orderings are considered, and also the insertion of magnesium as dopant is not able to alter the filling and the ordering of copper orbitals. The only noticeable difference between the pure and doped materials is that the sharp peaks in the DOS of copper atoms become slightly broader (see Figure SI1).

As for the electron population, formally in $\mathrm{KCuF}_{3}$ copper should be doubly positively charged and hence exhibit $\mathrm{d}^{9}$ population. The Mulliken population analysis on the computed wavefunctions assigns to the $\mathrm{d}$ shell of copper a population of about 9.3 electrons in the pure and the $\mathrm{Mg}$ doped crystals, which is consistent with the QTAIM outcome. The orbital population of copper does not depend on the spin ordering within the lattice, as we registered changes of about 0.01e among the different magnetic phases. The insertion of $\mathrm{Mg}$ into the crystal lattice and the relaxation of the position of fluorine atoms have no relevant effects onto the population of the d orbitals of copper, suggesting that (i) the perturbation due to $\mathrm{Mg}$ doping is extremely localized, (ii) slight modifications of the Jahn-Teller distortion around $\mathrm{Cu}$ have little effect on the orbital ordering, and therefore (iii) the Jahn-Teller distortion and the orbital ordering have different energy scales.
The orbital occupation of copper atoms implies that spin polarization around $\mathrm{Cu}$ is concentrated along the intermediate and short $\mathrm{Cu}-\mathrm{F}$ bonds, as can be appreciated in Figure 2 for the AF1 phase. In the doped material no relevant spin polarization is found at the $\mathrm{Mg}$ sites, while neighbouring copper atoms remain essentially unchanged (Figure 3, AF1 phase). It is worth noting that a small amount of spin polarization is found also on fluorine atoms, and it depends strictly on the spin of neighbouring $\mathrm{Cu}$ atoms. In fact, copper neighbours at $1.93 \AA$ in $a b$ planes induce a spin population of about $0.08 \mathrm{e}$ on fluorine; the two $\mathrm{Cu}$ neighbours at $1.99 \AA$ along $c$ induce a polarization of $0.04 \mathrm{e}$ each, and the effect due to $\mathrm{Cu}$ atoms at $2.26 \AA$ is negligible. As a result, in all magnetic phases fluorine atoms in $a b$ planes have a net alfabeta population of $0.08 \mathrm{e}$, whose sign is the same of the closest copper atom. Conversely, $\mathrm{F}$ atoms along $c$ have a zero net alfabeta population in $\mathrm{AF} 1$ and $\mathrm{AF} 3$, and twice $0.04 \mathrm{e}=0.08 \mathrm{e}$ in $\mathrm{F}$ and AF2. These simple rules apply also in the Mg-doped crystals, with the remark that no spin polarization comes from $\mathrm{Mg}$ atoms, as can be seen in Figure 3. Interestingly, these results are in agreement with LDA+U calculations [4], according to which $\mathrm{Cu}(3 \mathrm{~d})-\mathrm{F}(2 \mathrm{p})$ hybridization induces a finite spin density on the fluorine ions.

\section{Magnetic Coupling}

The magnetic coupling in pure and $\mathrm{Mg}$-doped $\mathrm{KCuF}_{3}$ has been investigated according to the Ising model $[37,38]$, which is derived from the phenomenological Heisenberg spin Hamiltonian for the isotropic interaction between localized magnetic moments. Its application to solid state systems is based on quite rough approximations, but it is widely used because it gives reliable results and has the advantage of relating the magnetic coupling parameters $J$ to quantities directly available from first principles computations. We refer the interested reader to $[20,39,40]$ for a thorough discussion on this subject and applications.

We assumed that in $\mathrm{KCuF}_{3}$ the magnetic Hamiltonian contains just two relevant terms, describing the superexchange interaction between first neighbouring $\mathrm{Cu}$ pairs in $a b$ planes and along the $c$ axis. The total energy difference between two magnetic phases can then be expressed using the simple formula:

$$
\begin{gathered}
E_{P H A S E 2}-E_{P H A S E 1}= \\
\cdot n
\end{gathered}
$$

where $E$ is the total energy per cell, $J_{i}$ are the magnetic coupling parameters, $S$ is the magnetic moment of $\mathrm{Cu}$ atoms (taken as the average between the values in the two phases), $\mathrm{z}_{\mathrm{i}}$ is the number of first neighbours of a single $\mathrm{Cu}$ atom changing spin from one phase to the other, and $n$ is the number of $\mathrm{Cu}$ atoms per cell. Considering the magnetic phases referred to as $\mathrm{F}, \mathrm{AF} 1, \mathrm{AF} 2$, and $\mathrm{AF} 3$, there are four magnetic transitions that allow directly computing $J_{a b}$ and $J_{c}$ :

$$
\begin{aligned}
E_{A F 1}-E_{F} & =2 \cdot J_{a b} \cdot S^{2} \cdot \mathbf{0} \cdot n+2 \cdot J_{c} \cdot S^{2} \cdot \mathbf{2} \cdot n \\
& =4 \cdot n \cdot J_{c} \cdot S^{2}
\end{aligned}
$$


TABLE 4: Magnetic coupling parameters between $\mathrm{Cu}$ pairs in ab planes $\left(J_{a b}\right)$ and along the $\mathrm{c}$ axis $\left(J_{c}\right)$.

\begin{tabular}{lccc}
\hline Material & Transition & $J a b(\mathrm{~K})$ & $J c(\mathrm{~K})$ \\
\hline $\mathrm{KCuF}_{3}$ & $\mathrm{~F}-\mathrm{AF} 1$ & & -631 \\
\hline $\mathrm{KCuF}_{3}$ & $\mathrm{AF} 2-\mathrm{AF} 3$ & & -620 \\
\hline $\mathrm{KCuF}_{3}$ & $\mathrm{AF} 1-\mathrm{AF} 3$ & 17 & \\
\hline $\mathrm{KCuF}_{3}$ & $\mathrm{~F}-\mathrm{AF} 2$ & 7 & \\
\hline $\mathrm{KCu}_{0.875} \mathrm{Mg}_{0.125} \mathrm{~F}_{3}$ & $\mathrm{~F}-\mathrm{AF} 1$ & & -655 \\
\hline $\mathrm{KCu}_{0.875} \mathrm{Mg}_{0.125} \mathrm{~F}_{3}$ & $\mathrm{AF} 2-\mathrm{AF} 3$ & & -611 \\
\hline $\mathrm{KCu}_{0.875} \mathrm{Mg}_{0.125} \mathrm{~F}_{3}$ & $\mathrm{AF} 1-\mathrm{AF} 3$ & 8 & \\
\hline $\mathrm{KCu}_{0.875} \mathrm{Mg}_{0.125} \mathrm{~F}_{3}$ & $\mathrm{~F}-\mathrm{AF} 2$ & 28 & \\
\hline
\end{tabular}

$$
\begin{aligned}
E_{A F 3}-E_{A F 2} & =2 \cdot J_{a b} \cdot S^{2} \cdot \mathbf{0} \cdot n+2 \cdot J_{c} \cdot S^{2} \cdot \mathbf{2} \cdot n \\
& =4 \cdot n \cdot J_{c} \cdot S^{2} \\
E_{A F 3}-E_{A F 1} & =2 \cdot J_{a b} \cdot S^{2} \cdot \mathbf{4} \cdot n+2 \cdot J_{c} \cdot S^{2} \cdot \mathbf{0} \cdot n \\
& =8 \cdot n \cdot J_{a b} \cdot S^{2} \\
E_{A F 2}-E_{F} & =2 \cdot J_{a b} \cdot S^{2} \cdot \mathbf{4} \cdot n+2 \cdot J_{c} \cdot S^{2} \cdot \mathbf{0} \cdot n \\
& =8 \cdot n \cdot J_{a b} \cdot S^{2}
\end{aligned}
$$

In fact, in the phase transitions F to AF1 and AF2 to AF3 the magnetic coupling between $\mathrm{Cu}-\mathrm{Cu}$ neighbours in $a b$ planes remains unchanged, while the two neighbours of each copper atom directed along the $c$ axis change spin. The opposite happens for the AF1 to AF3 and the F to AF2 transitions, where the four first neighbours of each $\mathrm{Cu}$ atom in $a b$ planes change spin, while the ordering along the $c$ axis is preserved.

When $\mathrm{KCuF}_{3}$ is doped with $\mathrm{Mg}$, the copper atoms become inequivalent, and hence the magnetic coupling cannot be described just in terms of the two magnetic parameters $J_{a b}$ and $J_{c}$. However, as discussed in the previous section, the perturbation induced by $\mathrm{Mg}$ on the electronic properties of $\mathrm{Cu}$ atoms is extremely localized, and doping does not alter the properties of copper atoms nor the $\mathrm{Cu}-\mathrm{Cu}$ internuclear distances. We then applied also in $\mathrm{KCu}_{0.875} \mathrm{Mg}_{0.125} \mathrm{~F}_{3}$ the formulae used for the pure compound. We averaged the magnetic moment $S$ of all $\mathrm{Cu}$ atoms within the cell and considered just that the insertion of $\mathrm{Mg}$ into the lattice reduces the number of $\mathrm{Cu}-\mathrm{Cu}$ superexchange interactions as compared to the pure compound. The computed magnetic coupling parameters $J_{a b}$ and $J_{c}$ in $\mathrm{KCu}_{0.875} \mathrm{Mg}_{0.125} \mathrm{~F}_{3}$ are thus effective values averaged over all the $\mathrm{Cu}-\mathrm{Cu}$ interactions in the $a b$ planes and along the $c$ axis, respectively. The magnetic coupling parameters computed for pure and $\mathrm{Mg}$ doped $\mathrm{KCuF}_{3}$ at the B3LYP level of theory for the optimized structure are reported in Table 4.

It is well known that the evaluation of the magnetic coupling depends critically on the functionals adopted in the computation [41], and our computations confirm that a change in the percentage of Hartree-Fock exchange included in the Hamiltonian induces dramatic changes in the evaluation of $J$ terms. Even worse, literature studies prove that no systematic rule allows a priori defining the best functional for a given system. However, as will be discussed in the following, the B3LYP approach proves adequate for the materials under investigation in the present study.

First of all, B3LYP magnetic coupling parameters computed for the pure optimized structure compare well with the experimental data $[35,36,42-46]$, and to vary the HartreeFock exchange contribution associated with the BECKE/LYP functionals leads to largely inadequate results, which even fail to reproduce the correct order of magnitude of the $J$ terms. Data obtained on $\mathrm{KCuF}_{3}$ with these same exchange and correlation functionals but different Hartree-Fock exchange contributions are collected in Tables 1SI and 2SI of the SI section.

We also checked that, at the B3LYP level of theory, the ideal undistorted structure is not an energy minimum, and whatever small perturbation in the cubic lattice induces relaxation to the distorted minimum. An orbitally ordered solution has been obtained also for the undistorted lattice, and its energy is slightly smaller than the nonorbitally ordered one. Test computations conducted with completely different functionals, namely, the PBE and the $\mathrm{VBH}$ ones, foresee that orbital ordering is not complete even in the distorted structure (the population of the $\mathrm{d}_{\mathrm{z}^{2}}$ orbital decreases) and disappears when an undistorted geometry around copper atoms is applied. At the $\mathrm{VBH}$ level of theory the system is conducting not just in the cubic phase but also upon distortion of the lattice.

According to B3LYP results, the antiferromagnetic ordering of $\mathrm{Cu}$ spins along the $c$ axis is largely favoured, while the ferromagnetic ordering is preferred in $a b$ planes. Considering the $\mathrm{F}$ to AF1 transition or the AF2 to AF3 one gives very similar results for $J_{c}$, and the same happens for $J_{a b}$ determined with the AF1 to AF3 or the AF2 to F phase transition. The computed value for $J_{c}$ measures about $-600 \mathrm{~K}$, while the order of magnitude of $J_{a b}$ is $+10 \mathrm{~K}$. These data compare very favourably with first principles computations on this same material conducted by Moreira et al. [20], and they are also consistent with the experiments $[35,36,42-46]$ conducted on pure $\mathrm{KCuF}_{3}$ samples.

Magnetic coupling parameters obtained for the $\mathrm{Mg}$ doped material are very close to the values of the pure compound. The insertion of a single $\mathrm{Mg}$ atom on all 8 $\mathrm{Cu}$ sites clearly reduces the number of $\mathrm{Cu}-\mathrm{Cu}$ superexchange interactions, thus decreasing the energy differences between AF1 and F magnetic phases, in agreement with the experiment [26], but their average strength remains nearly unchanged. These findings suggest that a $12.5 \% \mathrm{Mg}$ doping of $\mathrm{KCuF}_{3}$ does not alter the fundamental mechanism and the magnitude of the interactions building up the magnetic ordering of copper spins within the structure. The magnetic coupling between copper atoms (and hence the energy difference between different magnetic phases) is therefore strictly related to their local environment, i.e., to the number of superexchange interactions between neighbouring $\mathrm{Cu}$ atoms, regardless of the presence of $\mathrm{Mg}$ as doping element. This result is consistent with previous literature data obtained with 
first principles cluster simulations of the pure compound $[47,48]$.

\section{General Discussion and Conclusions}

In this paper we investigated the electronic and magnetic properties of pure $\mathrm{KCuF}_{3}$ and of the $\mathrm{Mg}$-doped $\mathrm{KCu}_{0.875} \mathrm{Mg}_{0.125} \mathrm{~F}_{3}$ compound, by means of first principles periodic Density Functional computations. Tests conducted with various functionals indicated that the B3LYP scheme is adequate for these systems.

Optimized structural parameters for the pure compound agree well with experimental data. Conversely, our approach does not reproduce the observed crystal cell variations upon substitution of $\mathrm{Cu}$ with $\mathrm{Mg}$ atoms. A reason for such discrepancy might be the clustering of $\mathrm{Mg}$ atoms in real samples, while in our study they are regularly distributed within the lattice. The structural effect of $\mathrm{Mg}$ insertion is just a reduction of the anisotropy of $\mathrm{Mg}-\mathrm{F}$ distances upon relaxation.

Both in pure and doped compounds, we considered the four possible combinations of ferromagnetic (FM) and antiferromagnetic (AF) ordering between Next Nearest Neighbours $(\mathrm{NNN}) \mathrm{Cu}-\mathrm{Cu}$ pairs: the F, AF1, AF2, and AF3 phases. Our calculations correctly predict an insulating AF1 orbitally ordered ground state. Switching the FM interaction in the $a b$ plane into AF has a very limited energy cost, while a marked energy increase is found when the AF interactions along the $c$ axis are turned into FM ones. These energy differences reflect on the estimate of the magnetic coupling parameters $J_{a b}$ and $J_{c}$ computed according to the Ising model (about $+10 \mathrm{~K}$ and $-600 \mathrm{~K}$, respectively) and nicely agree with available experimental results. Doping with $\mathrm{Mg}$ has a very limited effect on the estimate of $J_{a b}$ and $J_{c}$. Overall, the properties of all atoms, with the exception of the six first neighbouring fluorine, seem to be unaffected by $\mathrm{Mg}$ insertion, suggesting that doping with $\mathrm{Mg}$ induces a strong but extremely localized perturbation. In particular, the bond properties, the absolute charges, and the electronic distribution in the $3 \mathrm{~d}$ orbitals of $\mathrm{Cu}$ ions $\mathrm{NNN}$ of $\mathrm{Mg}$ are identical to the pure $\mathrm{KCuF}_{3}$ compound. It is worth noticing that a complete orbital ordering of the $3 \mathrm{~d}$ electrons is retained in the doped sample even for $\mathrm{Cu}(\mathrm{a})$ ion (see Figure 4), despite the significant elongation of the shortest $\mathrm{Cu}(\mathrm{a})-\mathrm{F}$ bond from 1.93 to $1.99 \AA$. In other words the $\mathrm{OO}$ seems to be quite unaffected by structural distortions. This is in accord with the disentanglement of OO and cJTD foreseen by the model of Kugel and Khomskii [1]. Since Mg doping does not alter significantly the estimate of $J_{a b}$ and $J_{c}$, the energy ordering of the four magnetic phases is maintained, and the lowering of the energy difference between the AF1 and $\mathrm{F}$ phases can be explained just considering the reduced number of $\mathrm{Cu}-\mathrm{F}-\mathrm{Cu}$ superexchange interactions in the unit cell.

In agreement with previous literature, either in the pure or in the doped compound, the $3 \mathrm{~d}$ shell of $\mathrm{Cu}$ atoms is only partially filled, and residual spin polarization is found also on fluorine atoms. Copper atoms preferentially accumulate electron density along the two long $\mathrm{Cu}-\mathrm{F}$ bonds, while they exhibit a depletion along the four shortest $\mathrm{Cu}-\mathrm{F}$ bonds. The
$\mathrm{Cu}-\mathrm{F}$ interactions are dominated by the charge transfer from the metal to the halogen atom but retain a partially shared character, and hence $\mathrm{KCuF}_{3}$ should not be considered a purely ionic material. This is confirmed by the relatively small charge of the $\mathrm{Cu}$ ion $(+1.38 \mathrm{e})$, which is quite far from the formal value of $+2 \mathrm{e}$. $\mathrm{Mg}$ delivers a larger amount of electron density towards the neighbouring atoms as compared to $\mathrm{Cu}$, but this is almost entirely counterbalanced by neighbouring $\mathrm{F}$ atoms, which explains the moderate changes in the magnetic coupling parameters.

All the above findings converge in suggesting the extreme localization of the perturbation due to $\mathrm{Mg}$ doping in the $\mathrm{KCuF}_{3}$ system. The absence of long range effects, which should affect the physical properties of the hosting compounds in a more complex way, makes $\mathrm{Mg}$ a suitable dopant for the investigation of strongly correlated electronic systems by means of orbital dilution, and this is particularly true in $\mathrm{Cu}$ based compounds, due to the very similar ionic radii of $\mathrm{Cu}^{2+}$ and $\mathrm{Mg}^{2+}$ in octahedral coordination $(0.73$ and $0.72 \AA$, respectively).

\section{Data Availability}

All data relevant to this publication are included in the text and hence are available to everyone.

\section{Conflicts of Interest}

The authors declare that there are no conflicts of interest regarding the publication of this paper.

\section{Acknowledgments}

The authors gratefully acknowledge Dr. Davide Ceresoli for useful discussions

\section{Supplementary Materials}

Supplementary data include total and projected DOS of pure and $\mathrm{Mg}$-doped $\mathrm{KCuF}_{3}$ (Figure SI), projected density of states on copper atoms of pure $\mathrm{KCuF}_{3}$ (Figure SI), and magnetic coupling parameters between $\mathrm{Cu}$ pairs determined with different functionals and Hartree-Fock exchange contributions (Tables 1SI and 2SI). (Supplementary Materials)

\section{References}

[1] K. I. Kugel and D. I. Khomskii, "The jahn-teller effect and magnetism: Transition metal compounds," Soviet Physics-Uspekhi, vol. 25, no. 4, pp. 621-641, 1982.

[2] E. Pavarini, E. Koch, and A. I. Lichtenstein, “ Mechanism for Orbital Ordering in "' Physical Review Letters, vol. 101, no. 26, 2008.

[3] L. Paolasini, R. Caciuffo, A. Sollier, P. Ghigna, and M. Altarelli, "Coupling between spin and orbital degrees of freedom in KCuF3," Physical Review Letters, vol. 88, no. 10, pp. 10640311064034, 2002.

[4] N. Binggeli and M. Altarelli, "Orbital ordering, Jahn-Teller distortion, and resonant x-ray scattering in KCuF3," Physical Review B: Condensed Matter and Materials Physics, vol. 70, no. 8, pp. 1-85117, 2004. 
[5] M. Scavini, M. Brunelli, C. Ferrero, C. Mazzoli, and P. Ghigna, "Experimental estimation of the cooperative Jahn-Teller energy in orbitally ordered $\mathrm{KCu} 0.8 \mathrm{Mg} 0.2 \mathrm{~F} 3$ perovskite," The European Physical Journal B, vol. 65, no. 2, pp. 187-190, 2008.

[6] P. Ghigna, M. Scavini, C. Mazzoli, M. Brunelli, C. Laurenti, and C. Ferrero, "Experimental disentangling of orbital and lattice energy scales by inducing cooperative Jahn-Teller melting in $\mathrm{KCu}_{1-x} \mathrm{Mg}_{x} \mathrm{~F}_{3}$ solid solutions," Physical Review B: Condensed Matter and Materials Physics, vol. 81, no. 7, 2010.

[7] I. Leonov, D. Korotin, N. Binggeli, V. I. Anisimov, and D. Vollhardt, "Computation of correlation-induced atomic displacements and structural transformations in paramagnetic KCuF3 and LaMnO3," Physical Review B: Condensed Matter and Materials Physics, vol. 81, no. 7, 2010.

[8] C.-Z. Wang, D.-Y. Liu, H.-B. Tang, J. Liu, and L.-J. Zou, "Spin flop transitions under strong magnetic fields in orbital ordered KCuF3," Physica B: Condensed Matter, vol. 405, no. 5, pp. 14231427, 2010.

[9] E. Pavarini, "Lattice distortions in KCuF3: A paradigm shift?" Annalen der Physik (Leipzig), vol. 523, no. 10, pp. 865-866, 2011.

[10] J.-S. Zhou, J. A. Alonso, J. T. Han, M. T. Fernández-Díaz, J.-G. Cheng, and J. B. Goodenough, "Jahn-Teller distortion in perovskite KCuF3 under high pressure," Journal of Fluorine Chemistry, vol. 132, no. 12, pp. 1117-1121, 2011.

[11] J. C. T. Lee, S. Yuan, S. Lal et al., “Two-stage orbital order and dynamical spin frustration in KCuF3," Nature Physics, vol. 8, no. 1, pp. 63-66, 2012.

[12] V. Gnezdilov, J. Deisenhofer, P. Lemmens et al., "Phononic and magnetic excitations in the quasi-one-dimensional Heisenberg antiferromagnet KCuF 3," Low Temperature Physics, vol. 38, no. 5, pp. 419-427, 2012.

[13] S. Yuan, M. Kim, J. T. Seeley et al., "Inelastic light scattering measurements of a pressure-induced quantum liquid in $\mathrm{KCuF3}$," Physical Review Letters, vol. 109, no. 21, 2012.

[14] L. G. Marshall, J. Zhou, J. Zhang et al., " Unusual structural evolution in $\mathrm{KCuF}$," Physical Review B: Condensed Matter and Materials Physics, vol. 87, no. 1, 2013.

[15] D. Legut and U. D. Wdowik, "Vibrational properties and the stability of the KCuF3 phases," Journal of Physics: Condensed Matter, vol. 25, no. 11, 2013.

[16] V. V. Iglamov and M. V. Eremin, "Orbital order fluctuations in KCuF3," Optics and Spectroscopy (English translation of Optika i Spektroskopiya), vol. 116, no. 6, pp. 828-831, 2014.

[17] K. Bieniasz, M. Berciu, M. Daghofer, and A. M. Oleś, "Green's function variational approach to orbital polarons in $\mathrm{KCuF3}$," Physical Review B: Condensed Matter and Materials Physics, vol. 94, no. 8, 2016.

[18] K. Bieniasz, M. Berciu, and A. M. Oleś, "Orbiton-magnon interplay in the spin-orbital polarons of KCuF3 and LaMnO3," Physical Review B: Condensed Matter and Materials Physics, vol. 95, no. 23, 2017.

[19] R. Dovesi, V. R. Saunders, C. Roetti et al., CRYSTAL06 User's Manual, University of Torino, Turin, Italy, 2006.

[20] I. D. P. R. Moreira and R. Dovesi, "Periodic approach to the electronic structure and magnetic coupling in $\mathrm{KCuF} 3, \mathrm{~K} 2 \mathrm{CuF} 4$, and $\mathrm{Sr} 2 \mathrm{CuO} 2 \mathrm{Cl} 2$ low-dimensional magnetic systems," International Journal of Quantum Chemistry, vol. 99, no. 5, pp. 805-823, 2004.

[21] R. Dovesi, C. Roetti, C. Freyria-Fava, M. Prencipe, and V. R. Saunders, "On the elastic properties of lithium, sodium and potassium oxide. An ab initio study," Chemical Physics, vol. 156, no. 1, pp. 11-19, 1991.
[22] K. Doll and N. M. Harrison, "Chlorine adsorption on the $\mathrm{Cu}(111)$ surface," Chemical Physics Letters, vol. 317, no. 3-5, pp. 282-289, 2000.

[23] R. Nada, C. R. A. Catlow, C. Pisani, and R. Orlando, "An abinitio hartree-fock perturbed-cluster study of neutral defects in lif," Modelling and Simulation in Materials Science and Engineering, vol. 1, no. 2, pp. 165-187, 1993.

[24] L. Valenzano, Y. Noël, R. Orlando, C. M. Zicovich-Wilson, M. Ferrero, and R. Dovesi, "Ab initio vibrational spectra and dielectric properties of carbonates: Magnesite, calcite and dolomite," Theoretical Chemistry Accounts, vol. 117, no. 5-6, pp. 9911000, 2007.

[25] R. H. Buttner, E. N. Maslen, and N. Spadaccini, "Structure, electron density and thermal motion of KCuF3," Acta Crystallographica Section B: Structural Science, vol. 46, no. 2, pp. 131-138, 1990.

[26] C. Oliva, M. Scavini, S. Cappelli, C. Bottalo, C. Mazzoli, and P. Ghigna, "Melting of orbital ordering in KMgxCul- xF3 solid solution," The Journal of Physical Chemistry B, vol. 111, no. 21, pp. 5976-5983, 2007.

[27] C. Gatti, "Chemical bonding in crystals: new directions," Zeitschrift für Kristallographie - Crystalline Materials, vol. 220, no. 5/6, pp. 399-457, 2005.

[28] F. Cargnoni and M. Scavini, "Direct-space analysis of the electronic structure of the $\mathrm{YBa} 2 \mathrm{Cu} 3 \mathrm{O} 6$ and $\mathrm{YBa} 2 \mathrm{Cu} 3 \mathrm{O} 7$ crystals," Canadian Journal of Chemistry, vol. 80, no. 3, pp. 235-244, 2002.

[29] L. Bertini, F. Cargnoni, and C. Gatti, "Chemical insight into electron density and wave functions: Software developments and applications to crystals, molecular complexes and materials science," Theoretical Chemistry Accounts, vol. 117, no. 5-6, pp. 847-884, 2007.

[30] C. Gatti, TOPOND-98 User's Manual , CNR - CSRSRC, Italy, Milan, 1998.

[31] R. Bianchi, C. Gatti, V. Adovasio, and M. Nardelli, "Theoretical and Experimental (113 K) Electron-Density Study of Lithium Bis(tetramethylammonium) Hexanitrocobaltate(III)," Acta Crystallographica Section B: Structural Science, vol. 52, no. 3, pp. 471-478, 1996.

[32] A. I. Liechtenstein, V. I. Anisimov, and J. Zaanen, "Densityfunctional theory and strong interactions: orbital ordering in Mott-Hubbard insulators," Physical Review B: Condensed Matter and Materials Physics, vol. 52, no. 8, pp. R5467-R5470, 1995.

[33] M. D. Towler, R. Dovesi, and V. R. Saunders, "Magnetic interactions and the cooperative Jahn-Teller effect in KCuF3," Physical Review B: Condensed Matter and Materials Physics, vol. 52, no. 14, pp. 10150-10159, 1995.

[34] I. Leonov, N. Binggeli, D. Korotin, V. I. Anisimov, N. Stojić, and D. Vollhardt, "Structural relaxation due to electronic correlations in the paramagnetic insulator KCuF3," Physical Review Letters, vol. 101, no. 9, 2008.

[35] D. A. Tennant, R. A. Cowley, S. E. Nagler, and A. M. Tsvelik, "Measurement of the spin-excitation continuum in onedimensional KCuF3 using neutron scattering," Physical Review B: Condensed Matter and Materials Physics, vol. 52, no. 18, pp. 13368-13380, 1995.

[36] D. A. Tennant, S. E. Nagler, D. Welz, G. Shirane, and K. Yamada, "Effects of coupling between chains on the magnetic excitation spectrum of KCuF3," Physical Review B: Condensed Matter and Materials Physics, vol. 52, no. 18, pp. 13381-13389, 1995.

[37] L. J. De Jongh and A. R. Miedema, "Experiments on simple magnetic model systems," Advances in Physics, vol. 23, no. 1, pp. 1-260, 1974. 
[38] L. J. De Jongh and R. Block, "On the exchange interactions in some $3 \mathrm{~d}$-metal ionic compounds. I. The 180 ॰ superexchange in the 3d-metal fluorides XMF3 and X2MF4 (X=K, Rb, Tl; M=Mn, Co, Ni)," Physica B+C, vol. 79, no. 6, pp. 568-593, 1975.

[39] Y.-S. Su, T. A. Kaplan, S. D. Mahanti, and Y.-S. Harrison, "Crystal Hartree-Fock calculations for $\mathrm{La} 2 \mathrm{NiO} 4$ and $\mathrm{La} 2 \mathrm{CuO} 4$," Physical Review B: Condensed Matter and Materials Physics, vol. 59, no. 16, pp. 10521-10529, 1999.

[40] I. de P. R. Moreira and F. Illas, " Ab initio theoretical comparative study of magnetic coupling in ", Physical Review B: Condensed Matter and Materials Physics, vol. 55, no. 7, pp. 4129-4137, 1997.

[41] N. Wannarit, C. Pakawatchai, I. Mutikainen et al., "Hetero triply-bridged dinuclear copper(ii) compounds with ferromagnetic coupling: A challenge for current density functionals," Physical Chemistry Chemical Physics, vol. 15, no. 6, pp. 19661975, 2013.

[42] S. K. Satija, J. D. Axe, G. Shirane, H. Yoshizawa, and K. Hirakawa, "Neutron scattering study of spin waves in onedimensional antiferromagnet KCuF3," Physical Review B: Condensed Matter and Materials Physics, vol. 21, no. 5, pp. 20012007, 1980.

[43] S. Kadota, I. Yamada, S. Yoneyama, and K. Hirakawa, "Formation of one-dimensional antiferromagnet in $\mathrm{KCuF} 3$ with the perovskite structure," Journal of the Physical Society of Japan, vol. 23, no. 4, pp. 751-756, 1967.

[44] K. Iio, H. Hyodo, K. Nagata, and I. Yamada, "Study of Magnetic Energy in One-Dimensional S=1/2 Heisenberg Antiferromagnet KCuF3 by Optical Birefringence," Journal of the Physical Society of Japan, vol. 44, no. 4, pp. 1393-1394, 1978.

[45] M. T. Hutchings, E. J. Samuelsen, G. Shirane, and K. Hirakawa, "Neutron-diffraction determination of the antiferromagnetic structure of KCuF3," Physical Review A: Atomic, Molecular and Optical Physics, vol. 188, no. 2, pp. 919-923, 1969.

[46] S. E. Nagler, D. A. Tennant, R. A. Cowley, T. G. Perring, and S. K. Satija, "Spin dynamics in the quantum antiferromagnetic chain compound KCuF3," Physical Review B: Condensed Matter and Materials Physics, vol. 44, no. 22, pp. 12361-12368, 1991.

[47] I. D. P. R. Moreira, F. Illas, and D. Maynau, "Local character of magnetic coupling in ionic solids," Physical Review B: Condensed Matter and Materials Physics, vol. 59, no. 10, pp. R6593-R6596, 1999.

[48] I. D. P. R. Moreira and F. Illas, "Ab initio study of magnetic interactions in $\mathrm{KCuF} 3$ and $\mathrm{K} 2 \mathrm{CuF} 4$ low-dimensional systems," Physical Review B: Condensed Matter and Materials Physics, vol. 60, no. 8, pp. 5179-5185, 1999. 

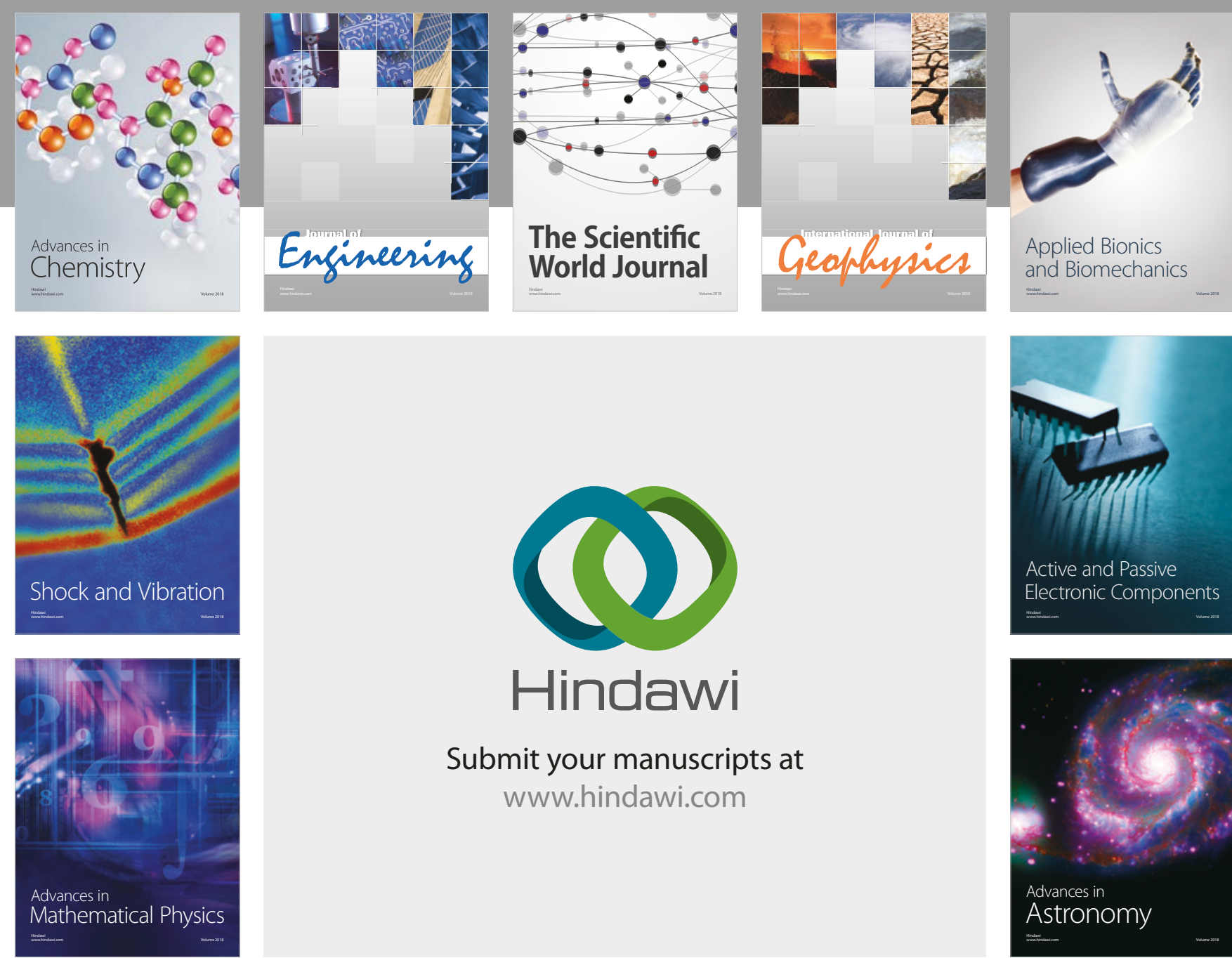

Submit your manuscripts at

www.hindawi.com

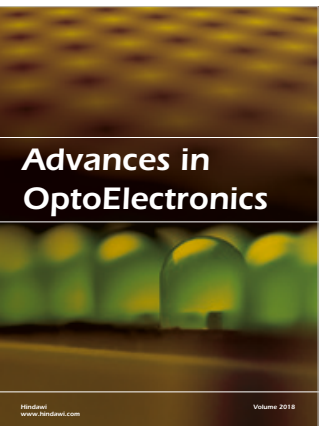

\section{Rotcting Machinery}
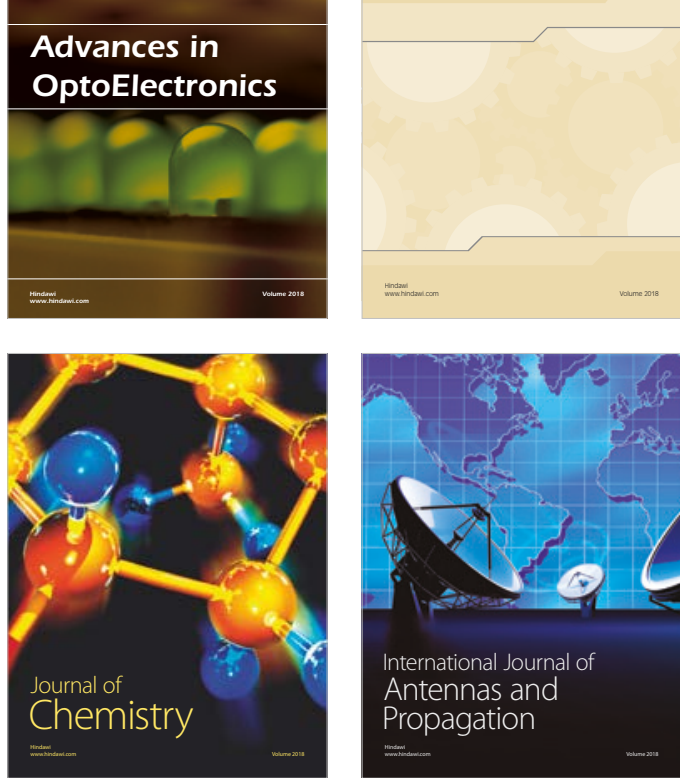

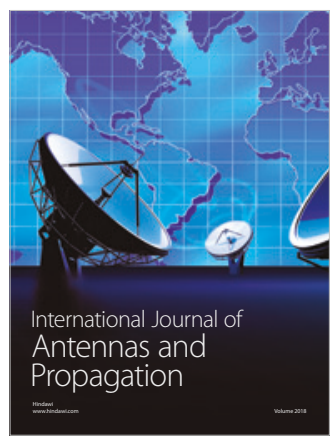

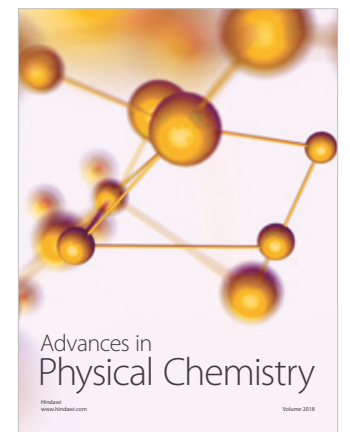

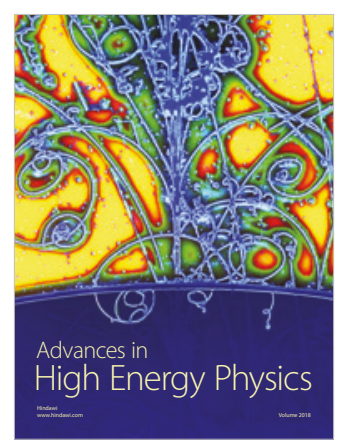

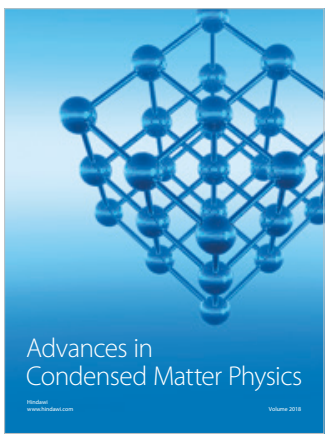

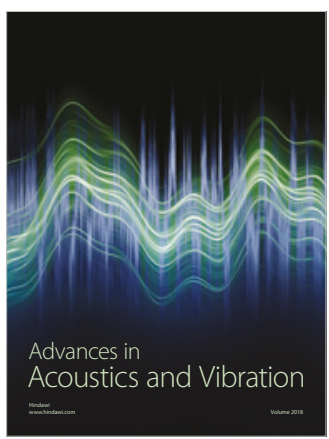

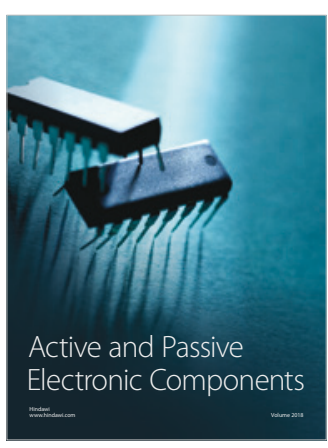
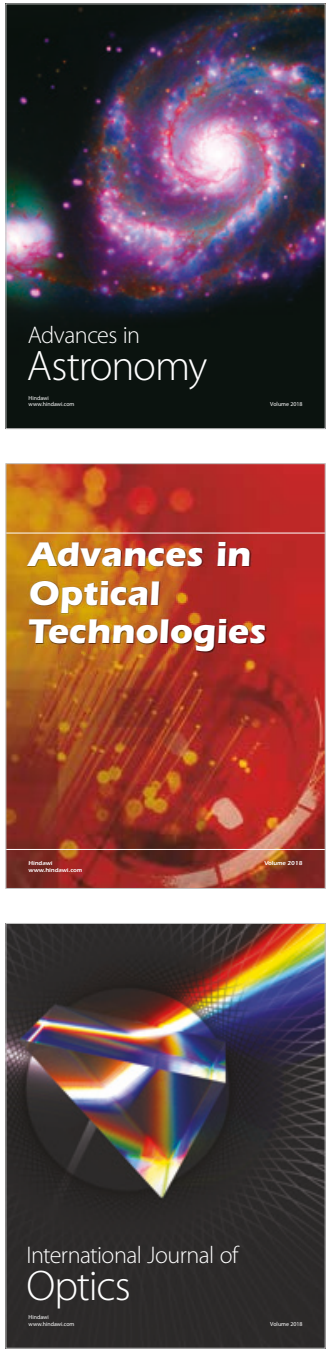